\title{
InspirerMundi-Remote Monitoring of Inhaled Medication Adherence through Objective Verification Based on Combined Image Processing Techniques
}

\author{
Pedro Vieira-Marques ${ }^{1}$ Rute Almeida ${ }^{1,2}$ João F. Teixeira ${ }^{3}$ José Valente ${ }^{4}$ Cristina Jácome ${ }^{1,2}$ \\ Afonso Cachim ${ }^{2}$ Rui Guedes ${ }^{2}$ Ana Pereira ${ }^{1,2,5}$ Tiago Jacinto ${ }^{1,4,6}$ João A. Fonseca ${ }^{1,2,4,5}$
}

${ }^{1}$ CINTESIS-Center for Health Technology and Services Research, Faculty of Medicine, University of Porto, Porto, Portugal

2 Department of Community Medicine, MEDCIDS, Health Information and Decision, Faculty of Medicine, University of Porto, Porto, Portugal

3 INESC TEC, Porto, Portugal

${ }^{4}$ MEDIDA-Serviços em Medicina, EDucação, Investigação,

Desenvolvimento e Avaliação, LDA, Porto, Portugal

${ }^{5}$ Allergy Unit, Instituto and Hospital CUF, Porto, Portugal

6 Department of Cardiovascular and Respiratory Sciences, Porto

Health School, Polytechnic Institute of Porto, Porto, Portugal
Address for correspondence Pedro Vieira-Marques, PhD, Faculty of Medicine, University of Porto, Al. Prof. Hernâni Monteiro, 4200 - 319 Porto, Portugal (e-mail: pmarques@med.up.pt).

\section{Abstract \\ Keywords \\ - medication adherence \\ - mHealth \\ - remote monitoring \\ - serious games}

Background The adherence to inhaled controller medications is of critical importance for achieving good clinical results in patients with chronic respiratory diseases. Self-management strategies can result in improved health outcomes and reduce unscheduled care and improve disease control. However, adherence assessment suffers from difficulties on attaining a high grade of trustworthiness given that patient self-reports of high-adherence rates are known to be unreliable.

Objective Aiming to increase patient adherence to medication and allow for remote monitoring by health professionals, a mobile gamified application was developed where a therapeutic plan provides insight for creating a patient-oriented self-management system. To allow a reliable adherence measurement, the application includes a novel approach for objective verification of inhaler usage based on real-time video capture of the inhaler's dosage counters.

Methods This approach uses template matching image processing techniques, an off-theshelf machine learning framework, and was developed to be reusable within other applications. The proposed approach was validated by 24 participants with a set of 12 inhalers models.

Results Performed tests resulted in the correct value identification for the dosage counter in $79 \%$ of the registration events with all inhalers and over $90 \%$ for the three most widely used inhalers in Portugal. These results show the potential of exploring mobile-embedded capabilities for acquiring additional evidence regarding inhaler adherence. received

May 19, 2020

accepted after revision

December 20, 2020

published online

March 27, 2021
DOI https://doi.org/

$10.1055 / \mathrm{s}-0041-1726277$

ISSN 0026-1270.

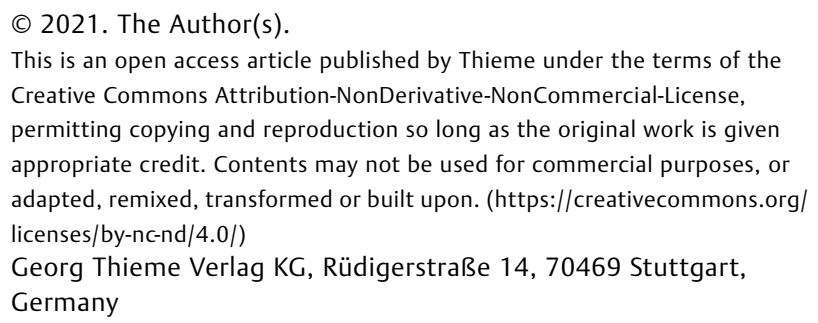


Conclusion This system helps to bridge the gap between the patient and the health professional. By empowering the first with a tool for disease self-management and medication adherence and providing the later with additional relevant data, it paves the way to a better-informed disease management decision.

\section{Introduction}

Incidence and prevalence of chronic diseases in general are increasing worldwide with a significant societal and financial impact. ${ }^{1}$ In the case of chronic respiratory disease which include asthma and chronic obstructive pulmonary distress (COPD) among others, evidence points to more than 1 billion individuals worldwide suffering from any of these conditions. This large incidence leads to an increased economic burden $^{2}$ which in Europe amounts to a total annual cost of more than $€ 380$ billion., 3 For asthma in particular, an estimate points to a mean cost for each patient per year of $€ 1,700$ in Europe and $€ 2,800$ in the United States. ${ }^{5}$

Chronic obstructive respiratory diseases (CORDs) are high-burden chronic diseases affecting patients throughout the life cycle. CORDs are already the third leading cause of death worldwide 6 and have a considerable impact on patients' quality of life. The possibility of health deterioration in a patient with CORD is permanent, increasing the susceptibility to exacerbations, which can cause frequent hospital admissions. Thus, patients with CORD require regular medical check-ups and monitoring of their health status.

Asthma is a particular case of CORD that may appear from an early age and is characterized by a chronic inflammation of airways placing severe limitations on daily life and being sometimes fatal. ${ }^{7}$

At present, asthma has no effective cure, so efforts are aimed at reducing manifestations of the disease. Being a chronic disease, treatment relies on reaching a stable condition and then maintain the control of this status in an attempt to minimize future exacerbations. ${ }^{8}$ Additionally, it has been established that self-management strategies can result in improved health outcomes, particularly for those with chronic diseases. ${ }^{9}$ Pinnock et al showed that supported self-management for asthma can reduce unscheduled care and improve asthma control, without significantly increasing the total health care costs. ${ }^{10}$

Inhaled controller medications are the cornerstone of asthma treatment, with established benefits in decreasing the severity and frequency of symptoms and exacerbations. $^{8,11,12}$ Albeit this evidence and in line with other long-term treatments, treatment adherence rates in asthma are known to be low. ${ }^{13,14}$ This poor medication adherence and persistence can be associated with increased rehospitalization, morbidity, mortality, and increased health care costs. $^{15}$

In this scenario, and for health professional's intervention to be effective and timely, monitoring of medication adherence plays an important role in keeping the disease under control. However, adherence evidence gathering suffers from difficulties in attaining a high grade of reliability ${ }^{16}$ creating the conditions for incorrect estimation of medication adherence. These difficulties can lead to several potentially expensive and dangerous problems such as effective treatments being judged as ineffective, expensive and unnecessary diagnostic procedures may be ordered, and changes in therapy that may be unnecessary or dangerously intensified. ${ }^{17}$ Furthermore, Jácome et al have reported a discordance in the assessment of inhaler adherence between health professionals and the patient. ${ }^{18}$ Being inhaler adherence currently a top priority for research and policy agendas, ${ }^{19}$ the need for objective adherence measures is reinforced to allow a better opportunity for improving patient-physician agreement and shared decision-making which is a relevant factor for medication adherence improvement. $^{20}$

Adherence evidence is mainly gathered through medical/dispensing records, or reliance on self-reports. ${ }^{21,22}$ Self-reports, although simple, cheap, and minimally intrusive are subjective and unreliable, nevertheless they are still considered one of the preferred methods to monitor adherence. $^{17,23}$

Some approaches developed to objectively measure adherence use, the sound produced during inhaler usage. ${ }^{24,25}$ But this implies attaching additional devices to the inhaler which may lead to a less integrated monitoring experience given that for instance they need to be recharged. Other approaches are based on electronic monitoring devices (EMD) designed for wrapping the inhaler and are integrated into the inhaler mechanical parts, allowing the evaluation of adherence rates by logging the time of actuation. These approaches are a step forward gaining some acceptance ${ }^{26-28}$; however, wide dissemination is difficult, costly, ${ }^{29}$ and usually do not allow easy access to acquire data and rely on the manufacturer proprietary system. These approaches although providing the advantage of objective readings, have currently limited feasibility for routine use in clinical practice and resource-constrained settings.

With the recognition that nonadherence is a major problem and that patient self-reports of adherence are unreliable, new objective adherence measurement tools need to be developed to provide additional evidence to better inform therapeutic decisions.

Survey data indicate that application-capable smartphone ownership and use are becoming quite common. According to the latest statistics, more than 3 billion people (almost half of the world population) are using a smartphone. $^{30}$

In this context, exploring ubiquitous and easy to use devices, packed with sensors, through mHealth approaches 
is highly promising and has good potential for delivering innovative adherence measurement tools easily integrated into everyday lives of asthma patients with asthma, promoting chronic disease management, and symptom control. ${ }^{31-33}$

The use of smartphones can be a relevant option to consider for changing behaviors that lead to improvements in asthma medication adherence. ${ }^{34,35}$ These may work in parallel to health professional's active intervention through enhancing information gathering directly or indirectly by the patient and the provision of automated personalized advice generated by the intervention based on information. ${ }^{36} \mathrm{Ad}-$ ditionally, gamification-based approaches, which consist on the use of game design elements in nongame situations, have been used with the purpose of making use of the potential motivating effect that games produce to increase the engagement in a particular activity. ${ }^{37}$ In the case of health care these approaches may evidence positive effect on health and wellbeing ${ }^{38}$ and have being used in asthma applications to motivate users. ${ }^{39}$

Inhaled controller medications are available through metered-dose inhalers (MDIs) and dry powder inhalers (DPIs). These inhalers are the main asthma medication intake devices in many countries. ${ }^{40}$ The more recent ones provide visual cues to the patient indicating the remaining dosages through numbers or scale representation. Although not revealing the effectiveness of each inhalation, these counters provide evidence on the actual use through activations count. ${ }^{41}$

As can be observed in - Fig. 1, different inhaler models and shapes are available in the market having different presentation approaches to dosage counters, evidencing large heterogeneity in dimensions and content (some provide sequential numbers, others number steps with pictographic indicators in between). Given the analogic nature of these counters, based on embedded mechanic mechanisms, to our knowledge, there is no straightforward way of gathering and processing these events through an automated approach.

Nevertheless, one common characteristic shared between inhalers is that counters are composed of text which retains evidence of inhaler usage, they are, however, located and shaped heterogeneously. In other areas of research, such as aids for the visually impaired, some approaches based on text detection techniques have been developed making use of smartphones for text extraction and text to speech translations from the context-acquired scenes and objects. ${ }^{42}$ In our research context, a therapeutic plan is available providing the necessary insight for a preassumption to be made regarding the particular inhaler shape to be used and a valuable expected pattern of use (number of inhalations per day in normal usage) that can foster a more accurate text detection operation.

Template matching is a classic and fundamental method in computer vision used to score similarities between objects for finding a subimage of a target image which matches a template image. ${ }^{43}$ This method is flexible and relatively straightforward to use.

Additionally, machine learning technology has been gaining wide adoption and popularity and pushed into mainstream software development by several application programming interfaces (APIs) being made available including pretrained models. Particularly for mobile devices, frameworks, like machine learning (ML) Kit from Google ${ }^{44}$ or Core ML from Apple, ${ }^{45}$ allow developers to include with staggering ease features for face detection, text detection, and object classification. ${ }^{46}$

A prototype of a novel approach to acquire inhaler's dosage counter progress, exploring the potential of mobile devices characteristics and available frameworks for image processing and machine learning-based text extraction, to acquire inhaler's dosage counter progress allowing for increased remote and objective monitoring was proposed as part of the application InspirerMundi. This approach was tested with a small subset of available inhalers and although showing promising preliminary results, ${ }^{47}$ needed improvements were also identified.

\section{Objective}

Thus, in this work, we aim to present the enhanced version of the inhaler usage detection module, designed having in mind the possibility of reuse in other applications, and the results of a laboratory validation study with all inhaler devices available in the Portuguese and Spanish markets.

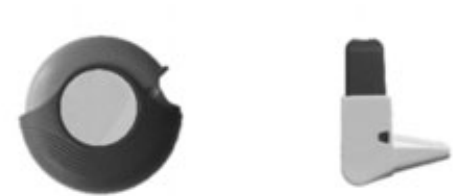

(A)

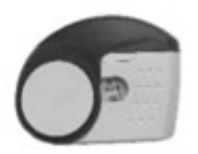

(G)

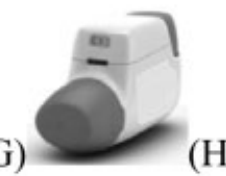

(H)

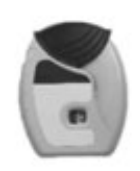

(B)

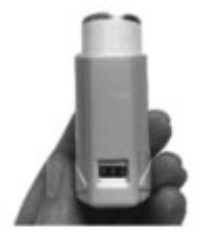

(C)

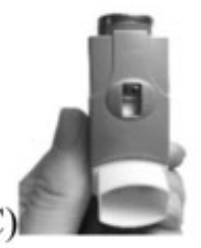

(I)
(D)

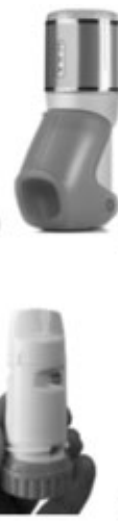

(K)

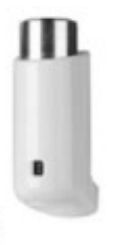

(F)

Fig. 1 Inhalers devices with numeric dose counters: (A) Diskus/Accuhaler, (B) Easyhaler, (C) Ellipta, (D) Flutiform MDI, (E) K-haler MDI, (F) 3M MDI, (G) Nexthaler, (H) Novolizer/Genuair, (I) Seretide MDI, (J) Spiromax, (K) Turbohaler, (L) Twisthaler 


\section{Methods}

\section{InspirerMundi Application}

The InspirerMundi application ${ }^{48}$ was designed with the intent of transforming the process of adherence to treatment into a positive experience through gamification and social interaction, while allowing for verified monitoring of treatment adherence. InspirerMundi' aims to support patients' medication management by providing an overall picture of the prescribed therapeutic plan and related reminders. It incorporates, a set of features in line with some of the solutions proposed to address different types of phenotypes in patient nonadherence ${ }^{19}$ (e.g., reminders, social support, and objective information acquisition).

It was developed through a highly iterative process incorporating input/feedback from patients and physicians throughout.

The application design and dynamics draw from social interaction and peer support the drive to prompt users to engage on a quest for maintaining the disease controlled and monitored while providing support to other community members. For that purpose, it includes, as the main interaction interface, a timeline in which expected events of monitoring and medication intake are depicted providing a quick reference when a medication is due (-Fig. 2 ).

The scheduling of events is in some cases predetermined, for example, for symptom and asthma control questionnaires (e.g., Control of Allergic Rhinitis and Asthma Test [CARAT] ${ }^{49}$ ) which have a fixed periodicity while in others, it is extracted from the therapeutic plan inserted by the patient. This plan includes the registration of the specific medications name, posology, frequency, and duration of the treatment (-Fig. 3 ). The health professional participation is also included by providing the user with the possibility of sharing its data with him/her at any moment (-Fig. 4). Besides planned medication, the user may also insert events of relief medication intake.

To promote user engagement, a gamification approach, was developed for wrapping the base functionalities and the process of medication detection and registration into a motivating experience.

Inspirers' driving story and dynamics derive from promoting users' social engagement in a quest for asthma medication adherence increase.

The main objective of the game is to increase the sphere of influence by coaching an expanding network of users that are designated as Warriors. Users start by assuming the role of a Warrior (beginner player) whose aim is to become an Inspirer (advanced player) to be able to socially influence other players. The Inspirer gives support to his/her Warriors promoting actively their evolution in medication adherence through social interaction.

Game mechanics are provided by a points system supporting users' progress throughout levels, making gradually
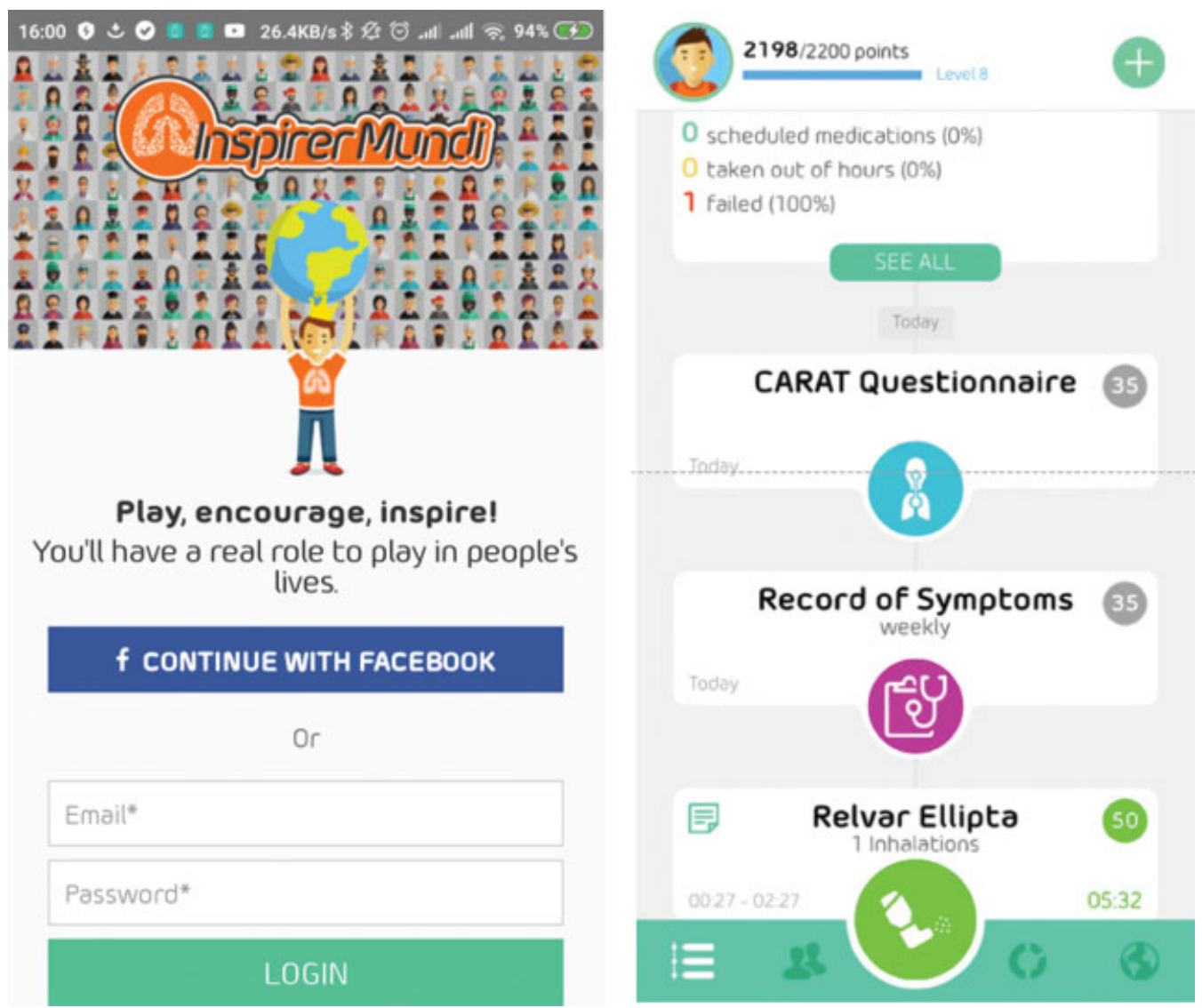

Fig. 2 Login and timeline. 
Step 1 Add medication menu

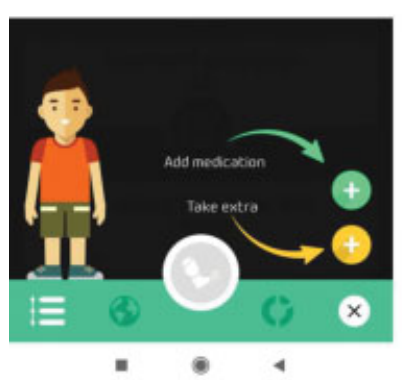

Step 2 Medication type and name

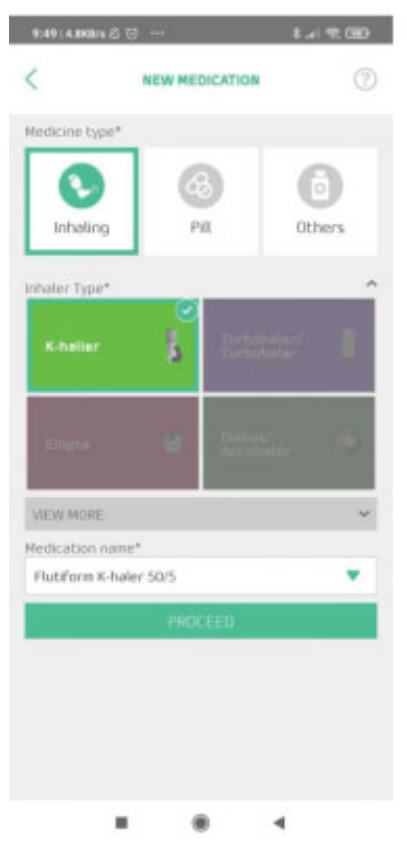

Fig. 3 Therapeutic plan input.

available new game interaction features, and user capabilities. The main components that support the game are points that are earned, when users take certain actions, such as register their medication intake at the right time, update medications, or get a positive assessment from other players in their network (Inspirer or Warrior). In addition, Inspirers may earn points from actions that promote their Warriors to earn points themselves (e.g., alerting that they should take their medication at the right time). This may be accomplished by exchanging messages and sending medication reminders through notifications. These features enable Inspirers to stimulate Warriors that are not compliant with the therapeutic plan, allowing for a more valuable network of influence.

Another encouragement for user's interaction are virtual badges, used to reward individuals with high application engagement. Whenever the user reaches a certain goal or does something special, he/she will get rewarded with a specific virtual badge. As an example, when an Inspirer becomes linked with his first Warrior, the user will get the badge "Role Model." These features along with game elements are illustrated in -Fig. 4.

\section{Inhaler Dosage Counter Detection Tool}

According to the scheduled events created from the inserted therapeutic plan (medication type and frequency), users are regularly prompted to register their medication intake. Scheduled medication events can be of three types: inhalation (referred throughout as inhalers), pills, and others (such as a nasal spray or an oral solution). When the event is for an inhaler the user can use the mobile phone to automatically acquire and register the inhaler counter value after the use. Upon notification of an inhaler scheduled event, the user opens the application and is prompted to confirm that he/she has taken the medication through the real-time inhaler dosage counter value identification module. This action is accomplished by presenting the inhaler to the smartphone camera that will automatically detect the inhaler and the inhaler counter value. Upon detection, the user is requested to validate the value detected.

The module uses the smartphone camera and provides, through image processing techniques and machine learning tools, confirmation of the inhaler presented to the camera and a dosage value inferred from the acquired dose counter image. This process depicted in - Fig. 5 happens in two phases that are described next.

\section{Template Matching}

The approach used to validate an inhaler is based on realtime template matching technique and is intended to confirm that the inhaler is presented to the camera in a certain position, which facilitates the identification of the dose counter location. Template matching is a digital image processing technique used to locate within an image, the area that best matches a certain object. This technique uses a source image and a template image where the source image is the space where a match with the reference template is searched for and the template represents the object that we intend to find. Template match algorithms perform the search by translation of the template image over the source image. However, for finding an object, using template matching, a few problems needed to be addressed, namely, the object's position, angle, and size.

We assume a given size and position such that it is comfortable for capture operation, guarantying the minimum resolution needed to later work on dial analysis of the intake counter. By choosing only one complete template per inhaler and not performing rotation matches, we are also restricting the acquisition position to a single orientation. This choice is enforced by displaying the inhaler template on screen.

Another factor to consider is how to distinguish a match from a nonmatch. Usually, this can be done by thresholding, although, as the images being acquired in this scenario usually include uncontrolled background, a single threshold would not suffice. That leads to a generation of a second threshold. Each template is searched for in the frame (source image) by correlation, Gaussian smoothing (for stabilizing locally maximal response), and selection of the most likely response position (the highest value). Afterward, these positions are evaluated on whether they are too far from, where 


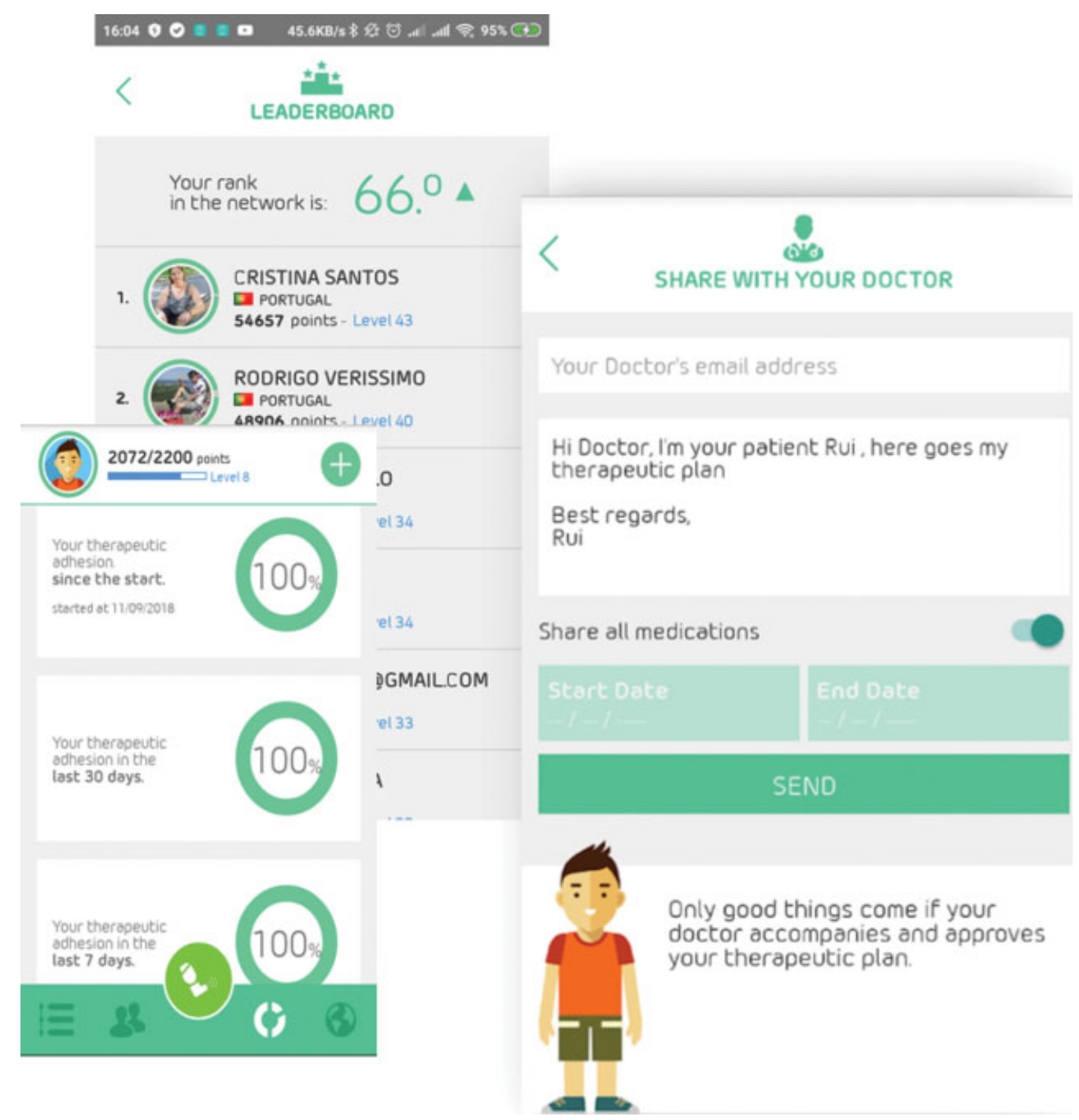

Fig. 4 Game achievements and adherence reporting and sharing screens.

they are expected to be, and if the distance between each other is within a tolerance range. The rationale is to combat spurious detection and guarantee that the inhaler is presented to the camera at the expected position and with the desired size.
As mentioned, two templates (-Fig. 6) are defined for each inhaler: a larger one representing the complete inhaler outline and a smaller one representing the dial Region of Interest (ROI) only. Both, are processed by invalidating highsaturation pixels, with a soft threshold masking over the HSV
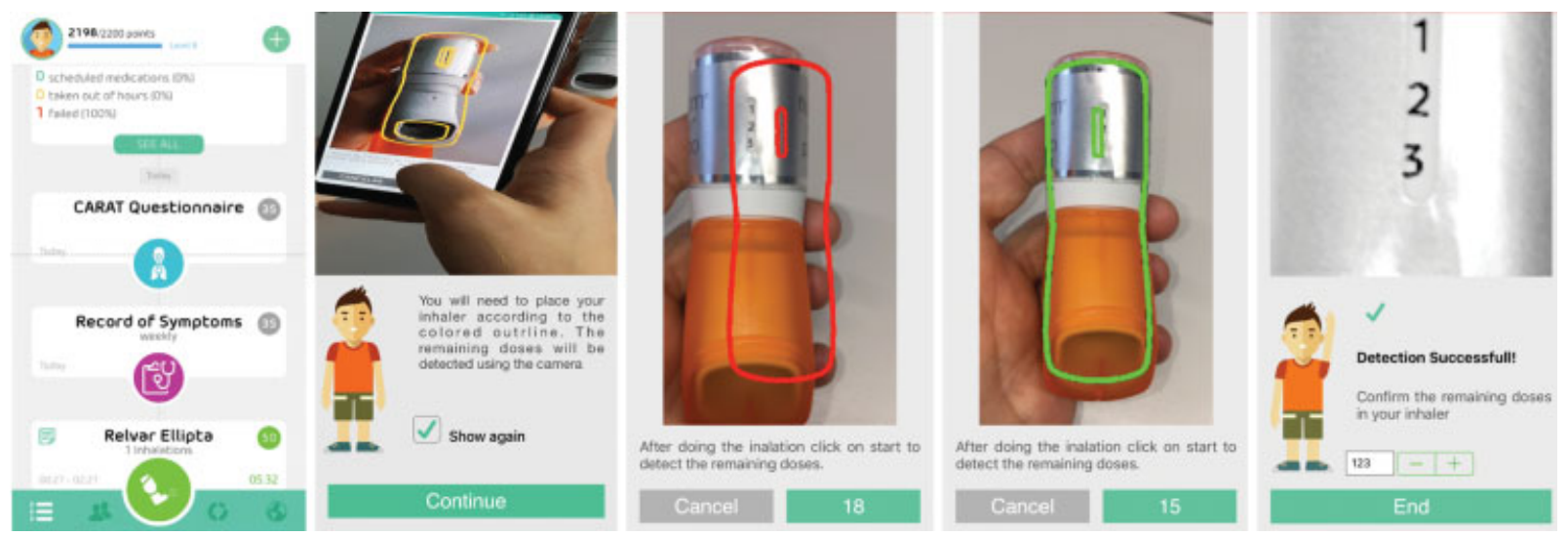

Fig. 5 Workflow for dosage detection counter. 

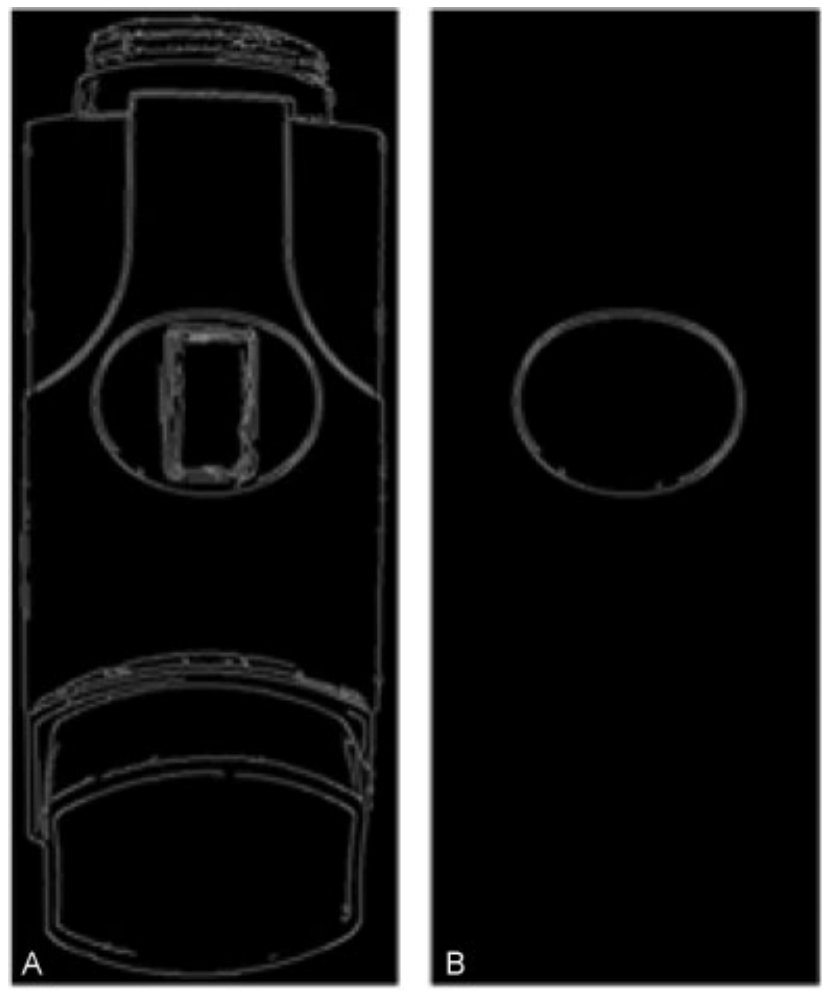

Fig. 6 Templates used for Flutiform MDI (A) full image (B) detail image.

(hue, saturation, value) space, performing morphological closing, and converting to greyscale. Subsequently, the edges of the objects are obtained, producing binary images. ${ }^{50}$ Each edge generally displays jagged lines and unstable contours, especially considering inhalers that present smooth surfaces that extend to the sides. As such, the representative edge maps are manually cleaned, removing spurious pixels and correcting notconnected lines, before being dilated and saved as templates.

Due to the variability of presentation of each inhaler device, some regions that are potentially simpler to detect, were manually removed from the template such as varying brand stickers with serial numbers or the numeric values on the intake display dials. On the other hand, due to the likeness between some dials to background objects or recurring geometric shapes, some inhaler dial templates (some of the smaller ones) include additional neighboring parts of the nondial inhaler edges as well.

\section{Dosage Counter Detection}

To address the challenge reading the dose counter, we use a ML-based tools. In particular, we use Google ML Kit that provides an off the shelf straightforward away for mobile developers to incorporate ML into applications without the need to fully master the whole process of training models. Aiming at providing objective verification of adherence, the inhaler detection module was extended incorporating the ML Kit text mining API for dosage counter value number extraction.

The module acquires, in the template matching phase, up to five frames were the outline detection occurred success- fully guarantying that the inhaler is present at an expected location. The module then processes each of the frames by first extracting a cropped image from the ROI. This region varies according to each inhaler, so a predefined section relative to the image size is defined. The image is then processed using the ML tool for the identification of blocks of text and the creation of a string representation from the extracted result. The module presents the output to the user based on the most frequent text detected among the set of successful frames acquired.

The detection module was developed as a complementary module to the application incorporating the necessary constructs to be easily reused within other applications.

For data processing and analysis, two additional corrections are performed after participants completed the tasks, outside the application and based on data stored for each of the frames collected. Correction no. 1 implied the elimination of all nonnumeric characters, as they are not present in any counter and result from insufficient/incorrect cropping or wrongly recognized characters. Correction no. 2 eliminated the nonacceptable numeric dose values by imposing an admissible interval. The admissible interval is defined by using the last confirmed dosage value (maximum) and an expected value (minimum) that is obtained by subtracting to the last confirmed value, the expected number of intakes that should have taken place between the last confirmed register and the actual date. In this simulation the actual value of the inhaler was used to determine the last recorded intake value (confirmed dosage value), assuming full adherence in the previous 2 days. A realistic therapeutic plan for each specific device inhaler was considered based on expert opinion. In both cases, the most common value after correction is chosen.

\section{Validation Study}

To validate the real-time inhaler dosage counter value identification module, a group of 24 participants was recruited within the university department staff, 10 were males and 14 were females with ages ranging from 20 to 55 years. Each participant was provided with a mobile device (iPhone 6s) and then asked to use the inhaler dosage counter value identification tool for registering a set of inhalers. All 12 device types having a numeric dose counter representing all the available devices in the Portuguese and Spanish market were tested (-Fig. 1).

For some types of inhalers, more than one device was available but all had different dosage counter numbers represented. Upon each inhaler identification procedure that did not present the user with a correct number, participants were asked to insert the correct number present in the inhaler.

A correct detection was defined by comparing the most frequent text detected among the set of successful frames acquired with the actual values visible in the dose counters. For cases in which several values were fully visible, any of those was accepted as correct if detected. Data from each detection event were collected and stored in the device for further processing. 
Table 1 Results obtained from the tests for all inhalers

\begin{tabular}{|l|l|l|l|l|l|l|}
\hline & $\begin{array}{l}\text { Inhaler devices } \\
\text { by type }\end{array}$ & $\begin{array}{l}\text { Detection } \\
\text { events }\end{array}$ & $\begin{array}{l}\text { Outline } \\
\text { detection }\end{array}$ & $\begin{array}{l}\text { Counter number } \\
\text { recognition }\end{array}$ & $\begin{array}{l}\text { Counter } \\
\text { number } \\
\text { recognition } \\
\text { after } \\
\text { correction no. 1 }\end{array}$ & $\begin{array}{l}\text { Counter } \\
\text { number } \\
\text { recognition } \\
\text { after } \\
\text { correction no. 2 }\end{array}$ \\
\hline & $n$ & $n$ & $n(\%)$ & $n(\%)$ & $n(\%)$ & $n(\%)$ \\
\hline Diskus/Accuhaler & 3 & 72 & $72(100)$ & $61(85)$ & $63(88)$ & $67(93)$ \\
\hline Easyhaler & 2 & 48 & $48(100)$ & $28(58)$ & $37(77)$ & $45(94)$ \\
\hline Ellipta & 3 & 72 & $72(100)$ & $38(53)$ & $55(76)$ & $65(90)$ \\
\hline Flutiform MDI & 2 & 48 & $48(100)$ & $30(63)$ & $34(71)$ & $37(77)$ \\
\hline K-haler & 3 & 72 & $70(97)$ & $32(44)$ & $33(46)$ & $47(65)$ \\
\hline 3m MDI & 1 & 24 & $24(100)$ & $20(83)$ & $20(83)$ & $22(92)$ \\
\hline Nexthaler & 1 & 24 & $24(100)$ & $21(88)$ & $22(92)$ & $24(100)$ \\
\hline Novolizer/Genuair & 2 & 48 & $48(100)$ & $43(90)$ & $44(92)$ & $44(92)$ \\
\hline Seretide MDI & 2 & 48 & $47(98)$ & $7(15)$ & $9(19)$ & $67(93)$ \\
\hline Spiromax & 3 & 72 & $72(100)$ & $55(76)$ & $44(92)$ & $71(99)$ \\
\hline Turbohaler & 2 & 48 & $47(98)$ & $40(83)$ & $4(17)$ & $44(92)$ \\
\hline Twisthaler & 1 & 24 & $24(100)$ & $4(17)$ & $432(70)$ & $4(17)$ \\
\hline Total (average) & 25 & 600 & $596(99)$ & $387(63)$ & & $488(79)$ \\
\hline
\end{tabular}

\section{Ethical Considerations}

The protocol was reviewed by the ethical committee from Hospital de São João and Faculty of Medicine of University of Porto and a waiver was obtained.

\section{Results}

In total, 600 events were recorded by all the 24 participants included in the validation study. All participants completed the designated task for all the inhalers made available and the obtained results are presented in -Table 1.

The results obtained reveal an overall good detection performance for every type of inhaler on the outline detection phase. In almost every case, the inhaler was detected at least in one frame and five frames, maximum limit was reached for $97 \%$ (582) of the events.

In the dosage counter, number identification phase based on the most common value, half of the inhalers revealed a correct dosage counter identification on over $75 \%$ of the events. On the other hand, two inhalers revealed a very low percentage of correct dosage counter identifications: Seretide MDI (15\%) and Twisthaler (17\%).

Applying correction no. 1, the overall performance increased in some cases by $20 \%$ (Easyhaler, Ellipta, and Spiromax inhalers).

With correction no. 2, there was also an increase in detection accuracy which in some cases was over 15\% (Easyhaler, Ellipta, K-haler, and Seretide MDI).

After both corrections, global performance reached an average of $79 \%$ for all the inhalers. Dose recognition was achieved for at least $90 \%$ of the events in two-thirds of the inhalers and near $100 \%$ of the events in some inhalers. These corrections did not allow any performance increase for Twisthaler.

\section{Discussion}

We present the results of a laboratory study designed to validate the real-time inhaler dosage counter value identification module included in the InspirerMundi application. This module supports an objective verification of inhaler usage, which may enable and enhance remote monitoring of patient adherence by a health professional. A large range of inhaler devices, with heterogeneous shapes and dosages, counter value presentation was used for testing purposes. In almost four-fifths of the registration events, it was possible to obtain a valid identification of the dosage counter. In particular, the performance reached over $90 \%$ for the three most common inhalers in Portugal during 2016 (Diskus/Accuhaler, Turbuhaler, and Spiromax), according to a retrospective population-based analysis made by the team in random sample of patients from the Portuguese electronic prescription and dispensing database (unpublished data and database described by Sá-Sousa et al, ${ }^{51}$ the analysis included all the tested inhalers but three that in 2016 were not available in the market: K-haler, Spiromax, and Ellipta). For the two inhalers with the lowest identification rates and after the inspection of the images used for text extraction, it was possible to identify that there were two different main reasons for failure. For Seretide MDI inhaler, an out of focus dosage counter display presented in most of the acquired images prevented a successful text recognition. This may be related to the used template size, which forced the inhaler to be too close to the camera, creating difficulties in the 
automatic focus process, along with a small size and deep dosage counter creating additional difficulties. Regarding Twisthaler, although the acquired images were of good quality, the adopted library with default trained model did not seem to be able to identify the characters, that is, the type of font present in the counter creates problems in the character classification. Twisthaler is rarely used in clinical practice, so the impact of the performance of the tool for this type of inhaler, in clinical practice, will be minimal.

We also observed that the detection method can be sensitive to highlight that create strong reflexes or shadows able to obscure the numbers. These effects prevent the acquisition of an image of enough quality to allow text detection, even for human eyes. The existence of an interface that allows the user to correct the identified value or to insert a value when identification was unsuccessful creates a failsafe system. Compared with the initial tests, the template matching phase performance increased substantially (from $42 \%$ over 5 inhalers ${ }^{47}$ to almost $100 \%$ over the 12 inhalers in the present study). The changes focused on redesigning inhaler templates by reducing graphical elements which allowed for a less strict match thus increasing the performance and the user experience. This course of action may lead to an increased chance of erroneous objects acquisition before the inhaler is presented to the camera. This is, however, minimized by the introduction of a delay on the actual acquisition of valid frames. Additionally, and despite that in this study, the images have been acquired in a more controlled environment, the increase in text recognition was also substantial, resulting from the increased performance in the acquisition of a valid frame.

Using template matching for inhaler type identification addressed the inexistence of trained models for inhaler recognition and classification which would allow a more flexible framing of the inhaler. The creation of a specific trained model for the available inhalers is the task ahead through which we expect to improve the lower performance devices and other issues.

In our opinion, with the obtained performance, it is reasonable to consider this method to be feasible for remotely monitor adherence to inhaled medication. The self-contained approach used for developing the module allows for easy integration on other applications where an objective measure of inhaler medication adherence is relevant.

This method is, however, still dependent on patient adherence to the application and the recording of inhalers. Nevertheless, the preference to engage in self-care through mobile technology is present in the literature where it is reported strong support for mHealth to assist asthma selfmanagement by both individuals with asthma and health care professionals. ${ }^{52-54}$

Additionally, serious games have been reported to have influence in promoting a healthy lifestyle and behavior change ${ }^{55}$; hence, we expect that the gamified approach included in the InspirerMundi application may promote additional user engagement developing its influence along two axes: (1) promoting the medication intake through reminders and increased patient awareness, and (2) a more objective registration of medication intake and disease control that can be easily shared with the health professional who may now take decisions regarding changes in the therapeutic plan with more strong evidence base. This approach is also in line with the identified potential to help a smoother transition from health professional supported to a more empowered self-management scenario.

\section{Conclusion}

The proposed method revealed to be feasible and promising for acquiring additional evidence regarding inhaler adherence that can be easily shared with the health professional remotely. This approach allows for exploring the potential of mobile device capabilities without the need for external devices or still expensive electronic monitoring devices.

To our knowledge, there are not many approaches proposed in the literature that make use of mobile devices and its imbedded sensors to gather effective activation count from inhaler devices, thus making this work relevant to help to mitigate the unreliability of patient adherence self-reports adherence. This system bridges the gap between the patient and the health professional by empowering the first with a tool for disease self-management and medication adherence promotion and creating the possibility of making available additional data to the last leading to better informed disease management decisions.

Funding

This work was funded by ERDF (European Regional Development Fund) through the operations: POCI-01-0145-36 FEDER-029130 ("mINSPIRE-mHealth to measure and improve adherence to medication in chronic obstructive respiratory diseases, generalization, and evaluation of gamification, peer support and advanced image processing technologies") cofounded by the COMPETE2020 (Programa Operacional Competitividade e Internacionalização), Portugal 2020 and by Portuguese Funds through FCT (Fundação para a Ciência e a Tecnologia).

\section{Conflict of Interest}

R.A. reports grants from FCT (Fundação para a Ciência e a Tecnologia), during the conduct of the study. No additional conflict of interest to be reported.

\section{Acknowledgment}

The authors would like to acknowledge all members of the INSPIRERS group (https://paceit.med.up.pt/sample-page/ inspirers-group/). They also acknowledge MundipharmaPortugal for supporting the dissemination of the InspirerMundi application.

\section{References}

1 Hajat C, Stein E. The global burden of multiple chronic conditions: A narrative review. Prev Med Rep 2018;12:284-293

2 GBD Chronic Respiratory Disease Collaborators. Prevalence and attributable health burden of chronic respiratory diseases, 19902017: a systematic analysis for the Global Burden of Disease Study 2017. Lancet Respir Med 2020;8(06):585-596 
3 European Respiratory Society. The economic burden of lung disease. Accessed September 18, 2020 at: https://www.erswhitebook.org/chapters/the-economic-burden-of-lung-disease/

4 European Respiratory Society. Respiratory Diseases in the World. Realities of Today - Opportunities for Tomorrow European Respiratory Society 2013

5 Nunes C, Pereira AM, Morais-Almeida M. Asthma costs and social impact. Asthma Res Pract 2017;3:1

6 GBD 2016 Causes of Death Collaborators. Global, regional, and national age-sex specific mortality for 264 causes of death, 19802016: a systematic analysis for the Global Burden of Disease Study 2016. Lancet 2017;390(10100):1151-1210

7 Bateman ED, Hurd SS, Barnes PJ, et al. Global strategy for asthma management and prevention: GINA executive summary. Eur Respir J 2008;31(01):143-178

8 Global Initiative for Asthma. Global strategy for asthma managment and prevention. Accessed September 18, 2020 at: https:// ginasthma.org/wp-content/uploads/2019/06/GINA-2019-mainreport-June-2019-wms.pdf

9 Taylor SJC, Pinnock H, Epiphaniou E, et al. A rapid synthesis of the evidence on interventions supporting self-management for people with long-term conditions: PRISMS - Practical systematic Review of Self-Management Support for long-term conditions. Southampton, UK: NIHR Journals Library; 2014

10 Pinnock H, Parke HL, Panagioti M, et al; PRISMS and RECURSIVE groups. Systematic meta-review of supported self-management for asthma: a healthcare perspective. BMC Med 2017; 15(01):64

11 O'Byrne PM, Barnes PJ, Rodriguez-Roisin R, et al. Low dose inhaled budesonide and formoterol in mild persistent asthma: the OPTIMA randomized trial. Am J Respir Crit Care Med 2001;164(8 Pt 1):1392-1397

12 Pauwels RA, Pedersen S, Busse WW, et al; START Investigators Group. Early intervention with budesonide in mild persistent asthma: a randomised, double-blind trial. Lancet 2003;361 (9363):1071-1076

13 Stanford RH, Averell C, Parker ED, Blauer-Peterson C, Reinsch TK, Buikema AR. Assessment of adherence and asthma medication ratio for a once-daily and twice-daily inhaled corticosteroid/longacting $\beta$-agonist for asthma. J Allergy Clin Immunol Pract 2019;7 (05):1488-1496.e7

14 Engelkes M, Janssens HM, de Jongste JC, Sturkenboom MC, Verhamme KM. Medication adherence and the risk of severe asthma exacerbations: a systematic review. Eur Respir J 2015;45(02): 396-407

15 Doz M, Chouaid C, Com-Ruelle L, et al. The association between asthma control, health care costs, and quality of life in France and Spain. BMC Pulm Med 2013;13:15

16 Wang PS, Benner JS, Glynn RJ, Winkelmayer WC, Mogun H, Avorn J. How well do patients report noncompliance with antihypertensive medications?: a comparison of self-report versus filled prescriptions Pharmacoepidemiol Drug Saf 2004;13(01):11-19

17 Lam WY, Fresco P. Medication adherence measures: an overview. BioMed Res Int 2015;2015:217047

18 Jácome C, Pereira AM, Almeida R, et al; Inspirers group. Patientphysician discordance in assessment of adherence to inhaled controller medication: a cross-sectional analysis of two cohorts. BMJ Open 2019;9(11):e031732

19 van Boven JFM, Lavorini F, Dekhuijzen PNR, Blasi F, Price DB, Viegi G. Urging Europe to put non-adherence to inhaled respiratory medication higher on the policy agenda: a report from the First European Congress on Adherence to Therapy. Eur Respir J 2017;49 (05):1700076

20 Wilson SR, Strub P, Buist AS, et al; Better Outcomes of Asthma Treatment (BOAT) Study Group. Shared treatment decision making improves adherence and outcomes in poorly controlled asthma. Am J Respir Crit Care Med 2010;181(06):566-577
21 Lima-Dellamora EDC, Osorio-de-Castro CGS, Madruga LGDSL, Azeredo TB. Use of pharmacy records to measure treatment adherence: a critical review of the literature. Cad Saude Publica 2017;33(03):e00136216

22 Garfield S, Clifford S, Eliasson L, Barber N, Willson A. Suitability of measures of self-reported medication adherence for routine clinical use: a systematic review. BMC Med Res Methodol 2011; $11: 149$

23 Kalichman SC, Amaral CM, Swetzes C, et al. A simple single-item rating scale to measure medication adherence: further evidence for convergent validity. J Int Assoc Physicians AIDS Care (Chic) 2009;8(06):367-374

24 D'Arcy S, MacHale E, Seheult J, et al. A method to assess adherence in inhaler use through analysis of acoustic recordings of inhaler events. PLoS One 2014;9(06):e98701

25 Taylor TE, Zigel Y, Egan C, Hughes F, Costello RW, Reilly RB. Objective assessment of patient inhaler user technique using an audio-based classification approach. Sci Rep 2018;8(01):2164

26 van Heuckelum M, van den Ende CHM, Houterman AEJ, Heemskerk CPM, van Dulmen S, van den Bemt BJF. The effect of electronic monitoring feedback on medication adherence and clinical outcomes: a systematic review. PLoS One 2017;12(10): e0185453

27 Pilcher J, Holliday M, Ebmeier S, et al. Validation of a metered dose inhaler electronic monitoring device: implications for asthma clinical trial use. BMJ Open Respir Res 2016;3(01):e000128

28 Chan AH, Harrison J, Black PN, Mitchell EA, Foster JM. Using electronic monitoring devices to measure inhaler adherence: a practical guide for clinicians. J Allergy Clin Immunol Pract 2015;3 (03):335-49.e1, 5

29 Howard S, Lang A, Sharples S, Shaw D. What are the pros and cons of electronically monitoring inhaler use in asthma? A multistakeholder perspective. BMJ Open Respir Res 2016;3(01): e000159

30 Statista.com. Number of smartphone users worldwide from 2016 to 2021. 2019. Accessed 18/09/2020

31 Hamine S, Gerth-Guyette E, Faulx D, Green BB, Ginsburg AS. Impact of mHealth chronic disease management on treatment adherence and patient outcomes: a systematic review. J Med Internet Res 2015;17(02):e52

32 Whitehead L, Seaton P. The effectiveness of self-management mobile phone and tablet apps in long-term condition management: a systematic review. J Med Internet Res 2016;18(05):e97

33 Bousquet J, Chavannes NH, Guldemond N, Haahtela T, Hellings PW, Sheikh A. Realising the potential of mHealth to improve asthma and allergy care: how to shape the future. Eur Respir J 2017;49(05):1700447

34 Liu WT, Huang CD, Wang CH, Lee KY, Lin SM, Kuo HP. A mobile telephone-based interactive self-care system improves asthma control. Eur Respir J 2011;37(02):310-317

35 Ryan D, Pinnock H, Lee AJ, et al. The CYMPLA trial. Mobile phonebased structured intervention to achieve asthma control in patients with uncontrolled persistent asthma: a pragmatic randomised controlled trial. Prim Care Respir J 2009;18(04):343-345

36 Morrison D, Mair FS, Yardley L, Kirby S, Thomas M. Living with asthma and chronic obstructive airways disease: Using technology to support self-management - an overview. Chron Respir Dis 2017;14(04):407-419

37 Deterding S. The lens of intrinsic skill atoms: a method for Gameful design. Hum Comput Interact 2015;30(3-4):294-335

38 Johnson D, Deterding S, Kuhn KA, Staneva A, Stoyanov S, Hides L. Gamification for health and wellbeing: a systematic review of the literature. Internet Interv 2016;6:89-106

39 Tinschert P, Jakob R, Barata F, Kramer JN, Kowatsch T. The potential of mobile apps for improving asthma self-management: a review of publicly available and well-adopted asthma apps. JMIR Mhealth Uhealth 2017;5(08):e113 
40 Ramadan WH, Sarkis AT. Patterns of use of dry powder inhalers versus pressurized metered-dose inhalers devices in adult patients with chronic obstructive pulmonary disease or asthma: An observational comparative study. Chron Respir Dis 2017;14 (03):309-320

41 Ibrahim M, Verma R, Garcia-Contreras L. Inhalation drug delivery devices: technology update. Med Devices (Auckl) 2015;8:131-139

42 Gupta N, Jalal AS. A robust model for salient text detection in natural scene images using MSER feature detector and Grabcut. Multimedia Tools Appl 2019;78(08):10821-10835

43 Banharnsakun A, Tanathong S. Object detection based on template matching through use of best-so-far ABC. Comput Intell Neurosci 2014;2014:919406

44 Machine learning for mobile developers Accessed September 18, 2020 at: https://developers.google.com/ml-kit/

45 Core ML: Integrate machine learning models into your app. Accessed September 18, 2020 at: https://developer.apple.com/documentation/ coreml

46 Çürükoğlu NMÖBuse. Deep Learning on Mobile Systems. Paper presented at: 2018 Innovations in Intelligent Systems and Applications Conference (ASYU) 2018; Adana, Turkey.

47 Vieira-Marques P, Teixeira JF, Valente J, et al. Combined ImageBased Approach for Monitoring the Adherence to Inhaled Medications. . Paper presented at: XV Mediterranean Conference on Medical and Biological Engineering and Computing - MEDICON 20192020; Coimbra.
48 Jácome CAR, Teixeira J, Vieira Marques P, et al. Inspirers: An app to measure and improve adherence to inhaled treatment. Paper presented at: International Conference e Health 2017 2017; Lisboa.

49 Fonseca JA, Nogueira-Silva L, Morais-Almeida M, et al. Validation of a questionnaire (CARAT10) to assess rhinitis and asthma in patients with asthma. Allergy 2010;65(08):1042-1048

50 Canny J. A computational approach to edge detection. IEEE Trans Pattern Anal Mach Intell 1986;8(06):679-698

51 Sá-Sousa A, Almeida R, Vicente R, et al. High oral corticosteroid exposure and overuse of short-acting beta-2-agonists were associated with insufficient prescribing of controller medication: a nationwide electronic prescribing and dispensing database analysis. Clin Transl Allergy 2019;9:47

52 Cook KA, Modena BD, Simon RA. Improvement in asthma control using a minimally burdensome and proactive smartphone application. J Allergy Clin Immunol Pract 2016;4(04):730-737.e1

53 Simpson AJ, Honkoop PJ, Kennington E, et al. Perspectives of patients and healthcare professionals on mHealth for asthma self-management. Eur Respir J 2017;49(05):1601966

54 Fonseca JA, Costa-Pereira A, Delgado L, Fernandes L, Castel-Branco MG. Asthma patients are willing to use mobile and web technologies to support self-management. Allergy 2006;61(03):389-390

55 DeSmet A, Van Ryckeghem D, Compernolle S, et al. A metaanalysis of serious digital games for healthy lifestyle promotion. Prev Med 2014;69:95-107 